\title{
Ocular traumatology: from military medicine to individual brilliance
}

\author{
Ferenc Kuhn
}

Received: 4 January 2013 / Accepted: 7 January 2013 / Published online: 23 January 2013

(C) Springer-Verlag Berlin Heidelberg 2013

In the current issue of Graefe's, the reader will find several interesting articles related to ocular traumatology.

There are two studies that have been performed using (different) national databases in the United States. One, by Armstrong et al., reviewed the National Electronic Injury Surveillance System All Injury Program to present the epidemiologic picture, during an 8-year period, of Emergency Department visits by children, defined as those under the age of 18 years.

They found that the rate of eye injury was highest in the 15to 17-year-old age group, with the most frequent cause to have been struck by or against an object. The study showed that, similarly to the findings in the adult population in most countries, the majority of the injuries occur at home (66\%). One would assume that the parents or a care-taker are at home most of the time, which would point to an obvious source of prevention. The fact that in this physically very active group only $9 \%$ of injuries occur while participating in sports is, as the authors point out, possibly due to prophylactic measures introduced many years ago - such as the face-guard in ice hockey. It is also likely that a high-profile success such as the dramatic drop in if not total elimination of serious eye injuries in ice hockey has a halo effect, and reinforces the need for prevention in other sports as well.

Using the National Electronic Injury Surveillance System to identify injuries caused by consumer products $(\mathrm{CP})$, the paper by Chen et al. surveys the other end of the age spectrum, injuries suffered by those 65 years and older. The youngest persons in the elderly age group (ages 65-69 years) had the

F. Kuhn $(\bowtie)$

International Society of Ocular Trauma, 2019 Hawkcrest Dr,

St. Johns, FL 32259-2971, USA

e-mail: fkuhn@mindspring.com

F. Kuhn

Helen Keller Foundation, Birmingham, Alabama, USA highest frequency (36\%) of eye injuries, with a gradual decline toward ages 85 years and above.

The authors demonstrate a well-known source of serious eye injury in the elderly population. As they wisely point out, "The furniture category of CP-related eye injuries is significant, because most of these injuries resulted from falls rather than the product itself." Those treating large numbers of patients with severe ocular trauma rather often encounter elderly people who got injured falling in the bathtub, slipping on a rug and hitting a doorknob with their eyeball, tripping on a slippery carpet covering a stairwell etc. The significance in all of this is the great potential for prevention. Removing clutter, fixing carpets to the floor, installing anti-slippery strips in bathrooms and bathtubs are simple, inexpensive, yet very effective ways to prevent such trauma; one just has to think about taking these measures before, and not after, an accident happens.

The study identified a rather unique source of injuries, the topical administration of the wrong material instead of the intended eye drops. This is again a rather obvious target for prophylaxis, especially if the application of medications can be supervised by someone.

Finally, Chen et al. correctly point out that it is much easier to prevent events that are task-oriented than those that are not. A relative with the correct mindset can go a very long way in organizing the environment surrounding elderly people who are at risk.

The papers by Eldaly et al. on the eye injury scene during Egypt's 2011 Revolution, and by Boiko et al. on the casualties in Russia's anti-terror operations in the Northern Caucasus, can by and large be categorized as military medicine. The injuries sustained are caused by weapons used with the intent to kill or maim and come in large numbers, even if the management had been done by civilian ophthalmologists in Cairo and military personnel in Russia. In Egypt, $73 \%$ of eyes had a presenting visual acuity of worse than 3/60; upon leaving the hospital, $77 \%$ had a vision worse than $1 / 60$. The severity of the (open-globe) trauma sustained during the 
armed conflict is shown in the Russian cohort by the fact that $19 \%$ of the eyes underwent primary enucleation - by a team that, correctly, emphasizes that "there is no reason for enucleation [even] in the case of low visual functions or even absence of light perception".

Interestingly, and obviously reflecting the modus vivendi of society in general, only $3 \%$ of those injured were females in Egypt. A full $77 \%$ of patients were admitted with the first $24 \mathrm{~h}$ of the injury in this country, and on average it took less than 6 days from being seen by an ophthalmologist to receiving the primary surgical intervention. One must assume that the time interval between injury and intervention (vitrectomy in case of posterior segment involvement, especially in eyes with open globe trauma) is crucial in terms of prognosis - which, interestingly, was not verified in the Russian study. The reason for this should be a focus of another investigative report.

Boiko et al. make an excellent point by suggesting that the Birmingham Eye Trauma Terminology (BETT) system should incorporate eyes that have been damaged "beyond recognition": a category of "eye destruction" needs to be added.

The article by Bauza et al. is technically not about war injuries; in reality, this paper on assault-related trauma from a tough New Jersey city is very similar due to the extreme severity of the injuries ("urban warfare"). The history itself is very revealing: glass bottles were the most common cause $(19.6 \%)$, followed by fists (16\%), powder and non-powder firearms (handgun, birdshot, and $\mathrm{BB}$ pellet, $10 \%$ ), and swinging objects (baseball bats, PVC piping, lead pipe, car club, and pool stick, $5 \%$ ).
The initial vision was no light perception (NLP) in $39 \%$ of eyes; among those with a known final visual acuity value, $46 \%$ of eyes had NLP vision, of which $31 \%$ of globes had been enucleated.

This very high rate of enucleation, especially in light of the studies from Egypt and Russia, again raises the question of how much the decision to remove the injured eye is a medicolegal, rather than medical, one in the USA.

Only $34 \%$ of eyes in the report by Bauze et al. underwent vitrectomy, as opposed to a $43 \%$ in the Russian and $50 \%$ (among eyes undergoing any type of secondary intervention) in Egypt. This also makes the reader wonder whether the lack of insurance influences a decision that otherwise should be purely medical.

Last but not least, the article by Forlini et al. shows the brilliance and creativity of an enthusiastic trauma specialist. This study presents the results in four patients of a novel surgical technique to overcome the challenges posed by the simultaneous presence of post-traumatic aphakia and aniridia. On a higher level, the results of a certain mindset is demonstrated by the report of Forlini et al. This surgeon (CF) is not satisfied by simply "following the crowd" and performing the type of surgery that all others would do in a similar situation, but designs a entirely new approach; he then carefully calibrates it to perfection, improving on the original idea based on the experience gained during the previous case/s. We all wish that more surgeons choose this path and continue answering the challenges of a seriously injured eye by approaching each case with an open mind and a mindset that continually seeks to find ever better solutions. 